\title{
HPV Typization in Vaccinated Women with Cervical Lesions
}

Jitka Kobikova ${ }^{1,3 *}$, Jan Evangelista Jirasek², Marcela Drazd'akova4, Miloslav Janousek ${ }^{1}$, Marie Strunova ${ }^{1}$, Vaclav Hejda ${ }^{2}$ and Jaroslav Horacek ${ }^{5}$

${ }^{1}$ Gynaecology and Obstetric Department, 1st Faculty of Medicine, Charles University, Prague, Czech Republic 'Institute for Mother and Child Care Prague 4, Czech Republic

${ }^{3}$ Centre for Gynaecological Oncological Prevention/COP/, Prague 4, Hrusická 2538, Czech Republic

${ }^{4}$ Laboratory for Sexual Deseases, 1 st Faculty of Medicine, Prague 2, Czech Republic

5 Institute for Pathology, Faculty of Medicine, OSLU, Ostrava, Czech Republic

\begin{abstract}
Background and objective: 24 patients, all of them vaccinated in postpubertal period, on their own descision, all of them had abnormal PAP tests. In all of them an expert colposcopy was performed, in all of them a low and high grade cervical lesion was detected. HPV genotypization, punch biopsy of the lesion folowed by histology and immunohistochemical staining of p16 INK4a were performed.
\end{abstract}

Study design: Our study concerns 24 patients suffering of cervical lessions. The age of these patients was 2034 years. 20 of them were vaccinated by Silgard and 4 of them by Cervarix.

Results: Two patients were hrHPV negative, in 21 patients the cervical lesions were infected with different HPV combinations, only in one woman single hrHPV type 52 was present. The frequent hrHPV types occurence was: $52 / 11 x /, 39 / 11 x /, 31 / 9 x /, 45 / 6 x /, 51 / 6 x /$.

Conclusions: Different high risk HPV combinations were present in 21 of 24 examined patients. The p16INK 4a positivity was detected in all lesions in all women examined.

Keywords: HPV vaccination; Cervical lesions; Cytology; Colposopy; p16ink 4a; HPV genotypization

\section{Introduction}

University Gynaecology Department cooperation with the cytology laboratory of the Centre for Gynaecological Oncological Prevention in Prague 4, Hrusická 2538, has inspired us by supply of significant material for assessment of inoculation results in the postpubertal period. That laboratory processes and evaluates screening PAP smears taken and performed by 28 district gynaecologists, who secure the gynaeological care at the area about 560,000 inhabitants, i.e. 200,000 women approximately. The dispatsch notes attached to the fixed cervical smears have shown the type of HPV vaccine used in 24 women having significant cevical cytological abnormalities of PAP smears and cervical lesions. The HPV vaccination time and data were given us by the district gynaecologists from the women health record. The aim of our study was to choose the optimal way for the proper care of the affected women. It was necessary to carry out complementary examinations to know tth more precise background of the cervical lesions in 24 women inoculated on teir own decisions in postpubertal period of their life

\section{Case Report}

The group of 24 patients with cervical cytological abnormalities of PAP smears were 20-24 years old. All of them were examined by expert colposcopists at the University Demartment. The colposcopy results were stated according to the "Barcelona 2" terminology which corresponds to the Bethesda systém 2001 cytology nomenclature. Cytology results are conveyed in Bethesda systém classification, i.e. ASC-US is atypia squamous cells of undetermined significance, ASC-H atypia squamous cells high grade lesion cannot be excluded, LSIL-low grade intraepitelial lesion corresponds with CIN 1 histology, HSIL cytological lesion corresponds to the CIN 2-CIN 3 on histology. In all enrolled women in our study, the Genotypisation test according to the LINEAR ARRAY HPV Genotyping Test. The punch biopsies of the women lesions were histologicaly verified and classified. After punch bipsies the tissue was stained in parafin sections/H-E/haematodxylin eosin procedure. The p16 ink4a visualised the abnormal and pathological tisue parts, using the GINtec p16ink 4a DAKO.

\section{Results}

The results are summarised in the Tables 1 and 2. Typical methods for examination of cervical lesions are demonstrated in colour pictures of two patients in Figure 1. Several methods have to be used to elucidate uncertain cases and make the exact diagnosis.

The p16 positive lesions were clasified histologicaly as CIN 1/22 patients/and CIN2/2 patients/condyloma planum 3x, condyloma inversum $2 \mathrm{x}$, condyloma accuminatum $1 \mathrm{x}$, acanthotic condyloma $1 \mathrm{x}$.

Regarding HPV, monotypic viral infection/hrHPV type 52/ was present in a single patient. In all remaining there were HPV combinations. Infection with two types of hrHPV was found in 10 women. Tritypic combination of HPV was present in 7 cases. Tetratypic combination was present in 2 patients. The viruses of the low risk category, 66, CP6108 were found in 3 women together with the high risk types. HPV negative were the cervical lesion in 2 cases.

\section{Discussion}

Regarding cytology, ASCUS is the most common atypia, affecting

*Corresponding author: Jitka Kobilkova, Gynecology and Obstetrics University Professor, DrSc, Fellow of International Academy of Cytology, 1st Faculty of Medicine, Charles University, Prague, Czech Republic, Tel: 420266081 111; E-mail: jitka.kobilkova@centrum.cz

Received January 20, 2014; Accepted February 24, 2014; Published February 26, 2014

Citation: Kobikova J, Jirasek JE, Drazd'akova M, Janousek M, Strunova M, et al. (2014) HPV Typization in Vaccinated Women with Cervical Lesions. J Cytol Histol 5: 223. doi:10.4172/2157-7099.1000223

Copyright: (c) 2014 Kobikova J, et al. This is an open-access article distributed under the terms of the Creative Commons Attribution License, which permits unrestricted use, distribution, and reproduction in any medium, provided the original author and source are credited. 


\begin{tabular}{|c|c|c|c|c|}
\hline & Age & Vaccine & Cytology & Colposcopy \\
\hline 1 & 1982 & Silgard & LSIL & LG \\
\hline 2 & 1990 & Silgard & ASC-H & LG \\
\hline 3 & 1984 & Silgard & ASC-H & LG \\
\hline 4 & 1990 & Silgard & LSIL & LG \\
\hline 5 & 1979 & Silgard & LSIL & LG \\
\hline 6 & 1982 & Silgard & ASC-US & LG \\
\hline 7 & 1988 & Cervarix & LSIL & LG + (inflam) \\
\hline 8 & 1986 & Silgard & LSIL & LG \\
\hline 9 & 1991 & Silgard & ASC-US & LG \\
\hline 10 & 1993 & Silgard & LSIL & LG \\
\hline 11 & 1989 & Silgard & ASC-US & LG \\
\hline 12 & 1978 & Silgard & ASC-H & LG \\
\hline 13 & 1988 & Silgard & ASC-H & LG \\
\hline 14 & 1984 & Silgard & ASC-H & LG + (PUS) \\
\hline 15 & 1979 & Cervarix & HSIL & $\mathrm{HG}(\mathrm{CIN} 2)$ \\
\hline 16 & 1985 & Silgard & ASC-H & LG \\
\hline 17 & 1983 & Silgard & ASC-H & LG \\
\hline 18 & 1976 & Cervarix & ASC-H & LG \\
\hline 19 & 1983 & Cervarix & LSIL & LG \\
\hline 20 & 1980 & Silgard & LSIL & LG \\
\hline 21 & 1984 & Silgard & HSIL & $\mathrm{HG}(\mathrm{CIN} 2)$ \\
\hline 22 & 1980 & Silgard & LSIL & LG \\
\hline 23 & 1988 & Silgard & ASC-H & LG \\
\hline 24 & 1990 & Silgard & LSIL & LG \\
\hline
\end{tabular}

Cervical Lesions and HPV Genotyping in Vaccinated Patients

Table 1: Vaccination and cervical lesions.

about $6 \%$ of the screened population in our country. ASCH a serious cytologic abnormality comprising $0.2-0.6 \%$ in screened population $[1,2]$. In our country the ASCH diagnosis is checked to $1 \%$ patients. In the CR the cytology result of ASCH is advised to undergo to colposcopy and biopsy in the same study [1]. The cells schowing metaplastic features, irregular nuclear borders, a mildly uneven chromatin distributio and/ or hyperchromazia [2] were evaluated as follows:

$56.5 \%$ of papillary lesions of the cervix were histologicaly classified as mature, $26 \%$ as immature, 17.3 as mixed. Corresponding smears were cytologicaly diagnosed as LSIL (6.2\%), ASCUS (7.3\%) and negative (10.4\%). Cylogy was not effective in detection of cervical condyloma accuminatum. Cytologic changes such as koilocytosis binucleation and dyskeratosis do not occure commonly and vaginal cytology appeared to underestimate. Condylomatous metaplastic lesions of the cervix changes are often associated, mature lesions with a lack of significant cytologic abnormalities and therefore with the diagnosis of ASCUS, or as negative missed.70\% of cytology negative cases and 37\% cases diagnosed as ASCUS were proven to be mature condyloma on histology [1].

The overal prevalence of HPV infection has increased over past 20 years [2]. But relative prevalence of HPV enotypes has remained stabile over time, despite certain geographical differences. HPV testing is frequently performed in conjunction with PAP test in atypical and abnormal smears. HPV testing in primary screening has a lower predictive value [3]. New episodes of desease re either recurence or asynchronic multifocal lesion. This may account for the rare of viral genomic intergration of the infected cells. Integration of the DNA with the Genomic DNA is the most important stage in development of the HPV related cervical carcinoma [3]. There is no doubt that in cervical epitelium the pl6ink 4a expression is linked with HPV infection to preparative changes. Our experience is the same as the presence of p16ink4a has significant clinical importance as a biomarker of cervical epitelium proliferation detecting viral presence. The detection of expression could identify clinicaly important cases of HPV infection with a risk of progression toward dysplasia and carcinoma [4].

Two cases in our group of patients were hrHPV genotyping negative. The commonly recognized cancerogennic hrHPV, types of HPV 16 and 18 , were present in 4 our cases in combination with hrHPV 39, 51, 52. These patients were 33-34 years old therefore it is supposed that these patients were vaccinated to late. At the time of vaccination they were allready infected by the cancerogenic HPV. In the other remaining group/of 18 cases/, only one patient was infected with a single high risk HPV/HPV type 52/. In other 17 cases we found a combination of two types/9 cases/. High risk RHPV types in combination with a low risk HPV was present in 3 cases. The most frequent HPV types in the different combinations were hrHPV 52/11 cases/, hrHPV 39/10 cases/,

\begin{tabular}{|c|c|c|c|c|c|c|c|c|c|}
\hline \multicolumn{3}{|c|}{ Genotyping } & \multicolumn{2}{|c|}{ Biopsy } & \multicolumn{5}{|c|}{ p16 ${ }^{\text {ink-4a }}$} \\
\hline \multicolumn{3}{|l|}{31,45} & \multicolumn{2}{|c|}{ CIN 1} & & + & & & \\
\hline \multicolumn{3}{|l|}{33,52} & \multicolumn{2}{|c|}{ CIN 1} & & + & & & \\
\hline \multicolumn{3}{|l|}{ Neg. } & \multicolumn{2}{|c|}{ CIN 1} & & + & \multicolumn{3}{|c|}{ Condyloma Inversum } \\
\hline \multicolumn{3}{|l|}{31,39} & \multicolumn{2}{|c|}{ CIN 1} & & + & & & \\
\hline \multicolumn{3}{|c|}{$16,39,51,52$} & \multicolumn{2}{|c|}{ CIN 1} & & + & & & \\
\hline \multicolumn{3}{|l|}{ Neg. } & \multicolumn{2}{|c|}{ Immature met. } & & + & \multicolumn{3}{|c|}{ Condyloma akanthoticum } \\
\hline \multicolumn{3}{|l|}{$31,39,52$} & \multicolumn{2}{|c|}{ CIN 1} & & + & \multicolumn{3}{|c|}{ Condyloma planum } \\
\hline \multicolumn{3}{|l|}{33,45} & \multicolumn{2}{|c|}{ CIN 1} & & + & \multicolumn{3}{|c|}{ Condyloma planum } \\
\hline \multicolumn{3}{|l|}{$31,39,52$} & \multicolumn{2}{|c|}{ CIN 1} & & + & & & \\
\hline \multicolumn{3}{|l|}{52,66} & \multicolumn{2}{|c|}{ CIN 1} & & + & & & \\
\hline 18,39 & & & $\mathrm{CII}$ & $\sqrt{ } 1$ & & + & & & \\
\hline $31,39,51$ & & & $\mathrm{Cll}$ & $\sqrt{ } 1$ & & + & & & \\
\hline 33,45 & & & $\mathrm{CII}$ & $\sqrt{ } 1$ & & + & Con & dyloma planum & \\
\hline $39,31, \mathrm{CP} 6$ & 108 & & $\mathrm{CII}$ & $\sqrt{ } 1$ & & + & Condy & loma accuminatun & \\
\hline $18,52, \mathrm{CP}$ & 6108 & & $\mathrm{Cll}$ & $\sqrt{ } 2$ & & + & & & \\
\hline $31,45,52$ & & & $\mathrm{CII}$ & $\sqrt{ } 1$ & & + & & & \\
\hline 52,66 & & & CII & $\sqrt{ } 1$ & & + & & & \\
\hline $31,45,51$ & & & & $\sqrt{ } 1$ & & + & & & \\
\hline $33,39,45$ & & & $\mathrm{CIN}$ & $1-2$ & & + & & & \\
\hline $39,45,51$ & & & $\mathrm{Cll}$ & $\sqrt{ } 1$ & & + & & & \\
\hline 52 & & & CII & $\sqrt{ } 2$ & & + & Conc & dyloma Inversum & \\
\hline $16,39,51,5$ & & & & $\checkmark 1$ & & + & & & \\
\hline 31,51 & & & & $\sqrt{ } 1$ & & + & & & \\
\hline 39,52 & & & & $\sqrt{ } 1$ & & + & & & \\
\hline Vaccin & & Cytolog & ggy & Genotyp & oing & P. biop & psy & Remarks & \\
\hline Cervarix & $4 x$ & ASC-US & $3 x$ & 16 & $2 x$ & CIN1 & $20 x$ & C. planum & 3 \\
\hline Silgard & $20 x$ & ASC-H & $9 x$ & 18 & $2 x$ & CIN2 & $2 x$ & C. inversum & 2 \\
\hline & & LSIL & $10 x$ & 52 & $11 x$ & CIN1-2 & $1 x$ & C. akanthoticum & 1 \\
\hline & & HLSIL & $2 x$ & 39 & $10 x$ & & & C. accuminatum & 1 \\
\hline & & & & 31 & $9 x$ & & & & \\
\hline & & & & 45 & $7 x$ & & & & \\
\hline & & & & 51 & $6 x$ & & & & \\
\hline & & & & 66 & $2 x$ & & & & \\
\hline & & & & CP6108 & $2 x$ & & & & \\
\hline
\end{tabular}

Table 2: Vaccination and cervical lesions. 


\section{Colposcopy - cytology - p16 ${ }^{\text {INK4a }}$}

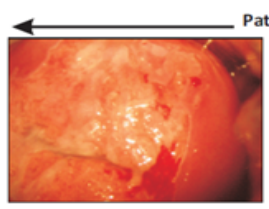

Colposcopy - low grade lesion

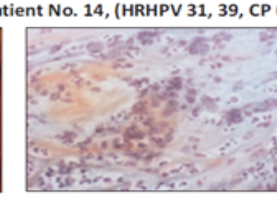

Cytology - ASC-H
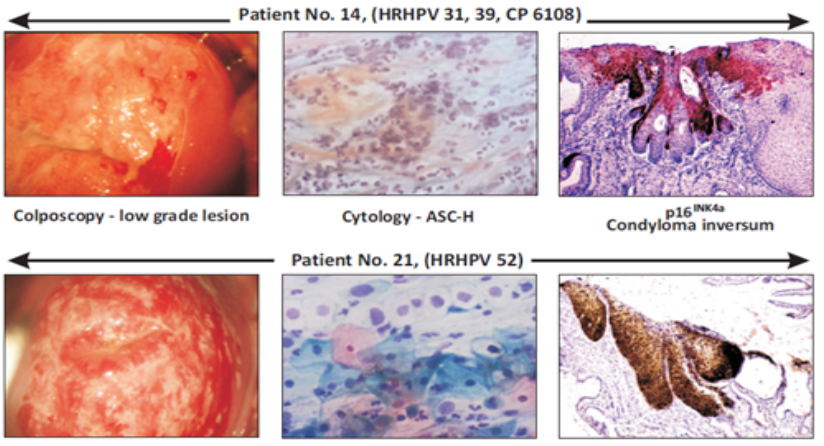

Colposcopy - low grade lesion
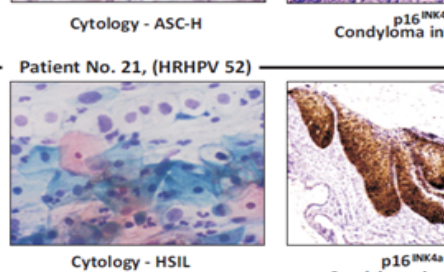
Condyloma inversum

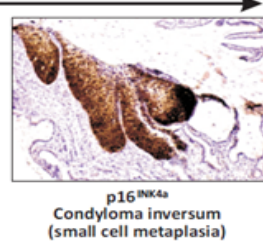

Figure 1: The Figure contains comparison of pictures of colposcopy, cytology, and immunohistology staining of the cervical tissue ( $\left.\mathrm{p} 16^{\text {ink4a }}\right)$ of the patient No. 14 and 21 in Table 1.

Patient No. 14: Cytology ASC-H: atypical squamous cells cannot HSIL exclude. Crowded sheet pattern, cells with degenerated enlarged slightly hyperchromatic nuclei, that show loss of polarity. The nuclei worrisome for a SIL with even contours. Many leucocytes, inflammation evidence. The chromatine irregularities are difficult to visualize. Histology result CIN1.

Patient No 21: Smear cells with metaplastic cytoplasm showing variations in size, shape, and ratio of nucleolar to cytoplasmatic area. On the right in picture there are isolated small cells with variable N/C ratio. Some cells displayed prominent nuclear irregulations. Koilocytes on the right picture corner. Follow up histology was CIN2.

hrHPV 31/9 cases/, hrHPV 45/6 cases/, hrHPV 51/6 cases/. The p16 INK4a positivity in cervical lesions of all followed women was found.

In two HPV negative patients the cervical lesions were classified histologicaly as condyloma inversum and acanthotic condyloma. There was a good correlation of the p16ink4a positivity and metaplastic changes classification. We suppose that the p16ink4a positivity coincides with a onset of viral infection. We suppose that the p16 ink4a positivity HPV might start in superficial located exhausted cylindrical glanduar cells, saving them from apoptosis. These infected cells escaping apoptosis may be conseqently reintroduced into the regenerating epithelium spreading the infection to the parabasal and basal regenerating cells of the metaplastic areas.

Regarding to use of vaccines, they are specifically focused and well working, providing a good protection regarding the cancerogenic hrHPV 16 and 18. Regarding the other HPV types, low risk HPV types such as 66, and CP 6108 it seems, that such infected lesions are of a short duration and that they heal spontaneously. Regarding lesions infected with all viral combinations with high risk HPV we do not know anything about their perspective for how long will they persist, how long and how will they change? Therefore, we conclude, that all cervical lesions infected with hrHPV viruses deserve long time clinical surveillance. The precise HPV genotyping and correlations with the behaviour in all cervical lesions is badly needed.

We found regarding the validity of our result we have to consider, that the population in our Republic is still almost white complexion. All our patients were Czech. Therefore, the frequency and viral combinations may be higly endemic. We would like to stimulate and initiate imillar studies in other countries. We do neither verestimate he validity of our results nor the small umber of ur patients.

\section{References}

1. Pinto AP, Calvaho MC, Kolb S, Tirone AF, Maia LR, et al. (2007) Value of Cytology in Papillary Condylomatous Lesions of the Cervix. Acta Cytologica 51: $51-60$

2. Galiano GE, Moatamed AN, Lee S, Salami S Apple SK (2011) Reflex High Risk HPV Testing in Atypical Squamous Cells Cannot Exclude High Grade Intraepitelial Lesion: A large Institutions Experience with the Significance of This Often Ordered Test. Acta Cytol 55: 167-172.

3. Sargent A, Bailey A, Almonte M, Turner A, Thomson C, et al. (2008) Prevalence of type-specific HPV infection by the age and grade of cervical cytology data from the artistic trial. Br J Cancer 98: 1704-1709.

4. Yoshida T, Sano T, Kanuma $T$, Inoue $H$, Itoh $T$, et al. (2011) Usefulness of CIN tec R Plus p16ink4a /Ki-67 Double-Staining in Cytological Screening of Cervical Cancer. Acta Cytologica 55: 413-420. 